\title{
Basal ganglia perfusion in the preterm infant during transition
}

\author{
Tim Schindler ${ }^{1,2}$, Yasmin Gilbert ${ }^{1}$, Sonali Jayatilake' ${ }^{1}$ Gordon Stevenson ${ }^{3}$, Ju Lee Oei ${ }^{1,2}$ and Alec Welsh ${ }^{1,4}$
}

BACKGROUND: The preterm brain is susceptible to changes in blood flow. Using power Doppler images, digital imaging techniques have been developed to measure the total amount of blood flow in a defined area, giving the index: fractional moving blood volume (FMBV). The aim of this study was to investigate temporal changes in basal ganglia perfusion during the transitional period after birth.

METHODS: Twenty-four preterm infants were examined with serial cranial ultrasounds at four time points during the first $48 \mathrm{~h}$ of life. FMBV was calculated using power Doppler images at each time point.

RESULTS: All infants had analyzable data and FMBV was successfully calculated at all time points. Twenty-three of the 24 infants had an increasing trend in FMBV over time. The median FMBV increased from $17 \%$ at $6 \mathrm{~h}$ to $25 \%$ at $48 \mathrm{~h}$. Oneway repeated measures ANOVA showed a significant increase in values at $P<0.001$ at each of the four time points.

CONCLUSION: We have demonstrated changes in basal ganglia blood flow as the cerebral circulation adapts to extrauterine life. With further investigation, this technique may be useful in the assessment of preterm circulatory adaptation, either alone or in conjunction with other modes of evaluating cerebral blood flow.

$\mathbf{T}$ he preterm brain is prone to changes in blood flow due to poor cerebrovascular autoregulation $(1,2)$. This is a wellestablished phenomenon that leads to fluctuations in cerebral blood flow with potentially devastating consequences for the developing brain (3). The immediate newborn period is therefore a vulnerable time for the preterm infant as surrogate markers of cerebral blood flow suggest that this is a period during which cerebral blood flow is variable and changing $(4,5)$. Low cerebral blood flow in early neonatal life has been shown to be an independent risk factor for intraventricular hemorrhage (IVH) and is associated with increased mortality and morbidity in preterm infants (6-8).

Direct measurement of regional cerebral blood flow and perfusion may provide important insights into the contributory factors leading to brain injury. One such novel technique of assessing regional cerebral perfusion in the neonate is to use the index: fractional moving blood volume (FMBV). Using Power Doppler ultrasound images, digital imaging techniques have been developed to measure the total amount of blood flow in a defined area. The measurements are expressed as a standardized index: FMBV (9). This technique has been validated and shown to correlate well with actual tissue perfusion in flow phantom studies and animal models $(10,11)$. Our research team has internally validated this technique in the neonate by demonstrating its reproducibility (12) and we have recently established a reference range for infants beyond the transitional period (13).

This study forms part of an investigation into the practicalities and clinical utility of this technique, and in this study, we aimed to investigate temporal changes in basal ganglia perfusion in the otherwise well preterm infant during the transitional period after birth. We hypothesized that an increase in regional perfusion would be observed in the first $48 \mathrm{~h}$ after birth.

\section{RESULTS}

During the study period, a total of 24 preterm infants between 25 to 35 wk gestation were recruited and cranial ultrasounds were performed at $6,12,24$, and $48 \mathrm{~h}$ after birth. None of the infants required inotropic support. Respiratory support varied at each time point from invasive and noninvasive modes of respiratory support to not requiring respiratory support. Germinal matrix IVH was documented on head ultrasound in three infants in this cohort. Bleeding was identified on the first head ultrasound in each of the infants at $6 \mathrm{~h}$ and did not progress in each case. No infants were withdrawn from the study. Physiological parameters known to affect cerebral blood flow were recorded where available and were within normal limits. The clinical characteristics of these infants including accepted normal physiological parameters are summarized in Table 1.

All newborns had analyzable data allowing estimation of basal ganglia perfusion by calculation of FMBV at each time point. The final FMBV was taken as the average of five calculations. Assessment of internal consistency showed an intraclass correlation coefficient of 0.89 (95\% CI: 0.85-0.92). The first image from each assessment was reanalyzed by a second observer and showed an intraclass correlation coefficient of 0.97 (95\% CI: 0.95-0.98).

\footnotetext{
'Faculty of Medicine, University of New South Wales, Sydney, Australia; ${ }^{2}$ Department of Newborn Care, Royal Hospital for Women, Sydney, Australia; ${ }^{3}$ Department of Engineering Science, University of Oxford, Oxford, England; ${ }^{4}$ Department of Maternal-Fetal Medicine, Royal Hospital for Women, Sydney, Australia. Correspondence:

Tim Schindler (tschindl@med.usyd.edu.au)

Received 1 March 2016; accepted 3 March 2016; advance online publication 15 June 2016. doi:10.1038/pr.2016.106
} 
Articles | Schindler et al.

Table 1. Clinical characteristics

\begin{tabular}{|c|c|}
\hline Clinical characteristics & Preterm infants $n=24$ \\
\hline GA at birth (weeks) & $31+5(25+4-35+5)$ \\
\hline Birth weight (grams) & $1,620(908-2520)$ \\
\hline \multicolumn{2}{|l|}{ Number ventilated (invasive) } \\
\hline $6 \mathrm{~h}$ & 6 \\
\hline $12 \mathrm{~h}$ & 1 \\
\hline $24 \mathrm{~h}$ & 1 \\
\hline $48 \mathrm{~h}$ & 1 \\
\hline \multicolumn{2}{|l|}{ Number ventilated (noninvasive) } \\
\hline $6 \mathrm{~h}$ & 13 \\
\hline $12 \mathrm{~h}$ & 16 \\
\hline $24 \mathrm{~h}$ & 12 \\
\hline $48 \mathrm{~h}$ & 9 \\
\hline \multicolumn{2}{|l|}{ No respiratory support } \\
\hline $6 \mathrm{~h}$ & 5 \\
\hline $12 \mathrm{~h}$ & 7 \\
\hline $24 \mathrm{~h}$ & 11 \\
\hline $48 \mathrm{~h}$ & 14 \\
\hline Mean arterial blood pressure $<\mathrm{GA}$ & 0 \\
\hline Arterial SaO2 $<90 \%$ & 0 \\
\hline Arterial pCO $2<35 \mathrm{mmHg}$ or $>50 \mathrm{mmHg}$ & 0 \\
\hline Temperature $<36.5^{\circ} \mathrm{C}$ or $>37.5^{\circ} \mathrm{C}$ & 0 \\
\hline Hemoglobin $<12 \mathrm{~g} / \mathrm{dl}$ or $>16 \mathrm{~g} / \mathrm{dl}$ & 0 \\
\hline PDA requiring treatment $<48 \mathrm{~h}$ & 1 \\
\hline Early onset sepsis & 0 \\
\hline Intraventricular hemorrhage & 3 \\
\hline No antenatal steroids $<35 \mathrm{wk}$ & 2 \\
\hline No magnesium sulphate $<30 \mathrm{wk}$ & 1 \\
\hline
\end{tabular}

Basic clinical characteristics with potential confounding clinical parameters and the incidence of additional factors that may have affected cerebral blood flow. Gestational age and birth weight are expressed as median (range). All other values are absolute numbers.

GA, gestational age; PDA, patent ductus arteriosus.

\section{Basal Ganglia FMBV}

FMBV increased over the first $48 \mathrm{~h}$ of life. Median FMBV rose from $17 \%$ at $6 \mathrm{~h}$ to $25 \%$ at $48 \mathrm{~h}$ (Figure 1 ). Twenty-three of the 24 infants had an increasing trend in FMBV over time. The FMBV increased from the $6 \mathrm{~h}$ to the $48 \mathrm{~h}$ time point in 22 of the 24 infants. FMBV data was normally distributed at each time point and is summmarized in Table 2.

There was a significant difference between mean FMBV values at the $<0.001$ level at the four time points $(\mathrm{F}(3,69)=13.77$, $P<0.001)$. Post-hoc comparison indicated that the mean FMBV at $6 \mathrm{~h}$ was significantly lower than the mean FMBV at 24 and $48 \mathrm{~h}$ and the mean FMBV at 12 and $24 \mathrm{~h}$ were both significantly lower than the mean FMBV at $48 \mathrm{~h}$. The differences between other time points were not significantly different.

\section{DISCUSSION}

Despite the importance of cerebral hemodynamics in the preterm infant, particularly during the transitional period shortly after birth, there is no readily available technique that reliably

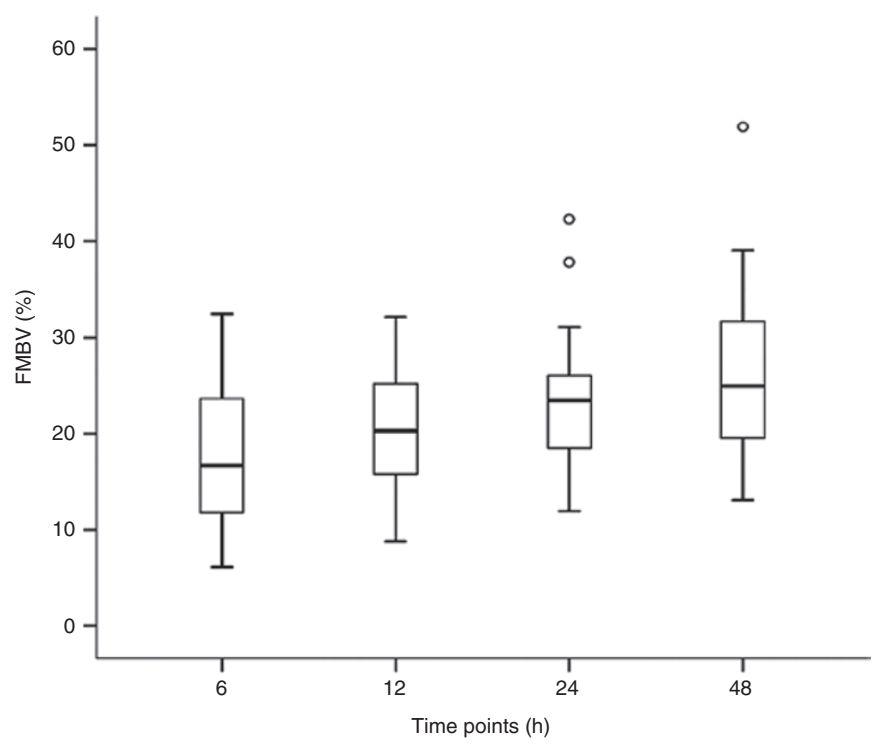

Figure 1. Fractional moving blood volume (FMBV) during transition. Box and whisker plot of FMBV at each time point. The box represents the interquartile range; the line in the box is the median and the bars are the range. Outliers are shown as open circles.

Table 2. FMBV over time

\begin{tabular}{lcccc}
\hline FMBV (\%) & $6 \mathrm{~h}$ & $12 \mathrm{~h}$ & $24 \mathrm{~h}$ & $48 \mathrm{~h}$ \\
\hline Median & 17 & 20 & 23 & 25 \\
Range & $6-32$ & $9-32$ & $12-42$ & $13-52$ \\
Mean & 18.1 & 20.7 & 23.3 & 26.8 \\
SD & 8.0 & 6.8 & 7.5 & 9.1 \\
\hline FMBV is conventionally expressed as a percentage. & & \\
FMBV, fractional moving blood volume.
\end{tabular}

quantifies regional cerebral blood flow or perfusion at the bedside. Diffuse optical spectroscopy, such as near-infrared spectroscopy, offers reliable trend monitoring of global cerebral oxygenation but does not measure potentially important changes in the regional distribution of blood flow $(14,15)$. Although superior vena cava blood flow, Doppler assessment of cerebral vessels, and cerebral oxygen saturations all have a sound theoretical basis for correlation with blood flow, these modalities are limited by being unable to directly measure blood flow in regions of interest. The technique FMBV offers a method of evaluating regional power Doppler signals, standardized using an area of $100 \%$ blood flow in the same region, to make measurements of regional cerebral blood flow that may be compared between infants.

The estimation of regional perfusion using power Doppler images has the potential to further the understanding of the pathophysiological changes that occur with respect to regional cerebral blood flow in infants at risk of neurological harm. The current understanding of cerebral blood flow during transition is based on assessment modalities that correlate with cerebral blood flow but do not directly measure it. This is the first study to measure blood flow in the brain during transition by directly examining a particular region of interest. By quantifying power Doppler signal using the index: FMBV, we have 
confirmed that there are transitional changes in regions of the brain that we know are vulnerable to injury (16).

We were able to successfully measure FMBV in all newborns at each time point. The accuracy of our image analysis was confirmed with excellent correlation between independent analyzers. There was good internal consistency between FMBV calculations at each assessment point; however, the correlation between values does not appear to be as strong as described in our reproducibility study (12). It is important to note that the internal consistency in this study reflects the entire image acquisition and analysis sequence whereas our earlier study focuses on the accuracy of image analysis only. The distribution of results at $48 \mathrm{~h}$ was reassuringly comparable with our recently established reference range (13).

The results of this longitudinal study show that basal ganglia perfusion in the preterm infant increased over the first $48 \mathrm{~h}$ of life. Given the overall clinical stability of this cohort of infants, it is likely that this transitional change in basal ganglia blood flow is a normal physiological process as the systemic blood flow increases. This is consistent with other studies investigating surrogate markers of cerebral blood flow during the transitional period $(4,17,18)$. This means that central regions of the brain, which are extremely sensitive to alterations in blood flow, follow a similar progression during the transitional period when compared to global cerebral blood flow.

We observed similar increases in basal ganglia perfusion at each time point. Given that the interval between examinations increased at each time point, this suggests that the rate of increase in blood flow per unit time decreased throughout transition. This is also consistent with transitional assessments using other modalities $(4,17,18)$. We noted that gestation did not appear to affect these transitional changes in regional blood flow although our numbers were too small to formally assess this. It is also important to highlight that this was a relatively mature cohort of appropriately grown infants and may not accurately reflect transitional changes in regional blood flow in extreme prematurity. It is possible that being clinically unstable during this period could result in extreme fluctuations in regional blood flow, which would then put the infant at risk of neurological harm.

The gold standard for assessing regional perfusion assessment is the use of labeled microspheres (19), which is not ethically acceptable or appropriate in newborn infants. It is pragmatically difficult to move preterm infants around, particularly in the immediate newborn period, to access modalities such as functional magnetic resonance imaging. In the absence of a similar bedside tool that evaluates regional perfusion, we were not able to externally validate our measurements of perfusion in our study design. Having demonstrated that there is a clear progression of FMBV during transition, it would be intuitive to now compare this technique with other surrogate markers of cerebral flow, such as near-infrared spectroscopy or SVC blood flow, on a larger scale to assess its clinical value.

There are some significant limitations to the quantification of power Doppler signal with respect to any region of interest. Selecting the region of interest accurately requires a high degree of precision as the color signal is sensitive to small changes in probe orientation. Consequently, regions have to be very carefully selected, requiring a well-defined plane of insonation with precise anatomical boundaries. In addition, many regions that would intuitively be of interest, for example, the periventricular region, do not have the detectable power Doppler signal required for a useful regional assessment of blood flow. The basal ganglia is a discrete structure with definable boundaries seen in a standard coronal view through the anterior horns of the lateral ventricles with the middle cerebral arteries in the same plane. Fortunately, the basal ganglia, which is often compromised in sick premature infants (20), has good power Doppler signal that can be demonstrated reliably using a standard plane of insonation.

In conclusion, we have shown that it is possible to measure FMBV as an estimation of basal ganglia perfusion in the preterm infant during transition. We have demonstrated changes in regional blood flow as the cerebral circulation adapts to extrauterine life. With further investigation, this technique may be useful in the assessment of preterm circulatory adaptation, either alone or in conjunction with more established modes of assessing cerebral blood flow.

\section{METHODS}

Between November 2012 and January 2014, a cohort of appropriately grown (10th - 90th percentile) preterm infants were recruited opportunistically, based on availability of study personnel and equipment, from the tertiary neonatal intensive care unit at the Royal Hospital for Women, Randwick, Australia. Inclusion criteria for the study were any preterm infant $<37 \mathrm{wk}$ gestation who was likely to be an inpatient for at least the first $48 \mathrm{~h}$ of life. Infants were excluded if they had a history suggestive of perinatal compromise (e.g., need for cardiac massage or adrenaline during delivery room stabilization, Apgar score $<7$ at $5 \mathrm{~min}$, cord $\mathrm{pH}<7.1$ ). Infants with known congenital anomalies that could affect cerebral blood flow were also excluded. Infants with minor germinal matrix IVH were included in the study. We prespecified that infants with major IVH would have been excluded from the analysis. Any infant who required inotropic support or became clinically too unstable to tolerate an examination would have been withdrawn from the study.

Ethics approval was obtained from the South Eastern Sydney Local Health District (Northern Sector) Human Research Ethics Committee (Reference Number: 11/104). Informed consent was obtained from parents or legal guardians prior to each scan.

\section{Ultrasound Imaging}

All ultrasound imaging was performed using a GE Voluson E8 ultrasound machine (GE Healthcare, Sydney, Australia) equipped with a 4-10 MHz wide band curved array transducer. The power Doppler ultrasound settings used in all examinations were: Quality "norm"; WMF "low1"; PRF "0.3kHz"; PD map "6"; Freq "low"; Smooth rise "10"; Smooth fall "4"; Flow res "mid1"; Line density "7"; Ensemble "11"; L filter "4"; Balance "190". "Sub-noise gain", the maximum Doppler gain level just below the presence of noise or blooming artefact, was used for all examinations $(21,22)$. The mechanical and thermal indices were always maintained below 1. A minimum of five consecutive good-quality images without movement artefact were stored digitally on the ultrasound machine's hard drive.

Images of the predefined region of interest within the neonatal brain were acquired through the anterior fontanelle. Specifically, the basal ganglia was imaged in a standard coronal view, bounded by the anterior cerebral artery medially, the middle cerebral artery inferiolaterally, and the lateral ventricle superiorly. All infants were examined with serial cranial ultrasounds during the first $48 \mathrm{~h}$ of life. 


\section{Articles $\mid$ Schindleretal.}

Power Doppler ultrasound was used to display regional blood flow. The amount of blood flow was quantified using the index FMBV, which involves using a large blood vessel in the area to determine $100 \%$ blood flow and evaluating the region of interest against this "maximum" blood flow. Cranial ultrasounds were performed on babies at $6,12,24$, and $48 \mathrm{~h}$. FMBV was calculated using power Doppler images at each time point.

Exportation of power Doppler images from the ultrasound machine was performed using the "Volume/Raw File" option. This provided a RAW format file, which stored power Doppler and gray scale data in a polar geometry $(\theta, r)$. Before FMBV analysis, an algorithm developed in Python (version 2.7.3) using the NumPy (23) and SciPy (24) libraries was used to convert the data to typical Cartesian geometry $(\mathrm{x}, \mathrm{y})$ images for user analysis. An application was developed by our group for the conversion of data and subsequent calculation of FMBV, adapted from the original description of the technique (9). This application is not yet freely available but we are working toward this.

The basal ganglia was identified and labeled using surrounding anatomical landmarks. The cumulative Doppler frequency shift was measured by counting the number and intensity of colour pixels within the region, inferring the number of moving blood cells and thus the total amount of blood flow. The cumulative power distribution function was used as originally described to correct for the effect of rouleaux, which scatter excessive ultrasound signal (11). Common confounding variables such as attenuation effects, field depth and machine settings are controlled by standardizing to a local value for $100 \%$ amplitude within the evaluated image. The standardized maximum value for blood flow was then used to correct regional perfusion measurements and calculate FMBV for the region of interest, the basal ganglia. The mean FMBV from five images was considered to be representative of the regional cerebral perfusion and is conventionally expressed as a percentage.

\section{Statistical Analysis}

Statistical analysis was performed using IBM SPSS Statistics 22 software (IBM, Armonk, NY).

The final FMBV is taken as an average of five calculations. The internal consistency was assessed by comparing these five values in all infants at all time points using an ICC. The first image from every assessment was reanalyzed independently by a second observer to ensure the accuracy of the analysis. The correlation between the two observers was assessed using an ICC.

Normality of data was assessed before analysis. One-way repeated measures ANOVA was used to compare FMBV values at each time point and then performed post-hoc comparisons between groups. A $P$ value of $<0.05$ was considered significant.

\section{STATEMENT OF FINANCIAL SUPPORT}

Running for Premature Babies (The Royal Hospital for Women Foundation, Randwick, Australia) supported this study through a research fellowship. GE Healthcare (GE Healthcare, Sydney, Australia) provided neonatal ultrasound probes for the conduct of the study.

Disclosure: The authors have no financial relationships relevant to this article to disclose. The authors have no conflicts of interest to disclose.

\section{REFERENCES}

1. Lou HC, Lassen NA, Tweed WA, Johnson G, Jones M, Palahniuk RJ. Pressure passive cerebral blood flow and breakdown of the blood-brain barrier in experimental fetal asphyxia. Acta Paediatr Scand 1979;68:57-63.

2. Lou HC, Lassen NA, Friis-Hansen B. Impaired autoregulation of cerebral blood flow in the distressed newborn infant. J Pediatr 1979;94:118-21.

3. Pryds O, Edwards AD. Cerebral blood flow in the newborn infant. Arch Dis Child Fetal Neonatal Ed 1996;74:F63-9.
4. Kluckow M, Evans N. Superior vena cava blood flow in newborn infants: a novel marker of systemic blood flow. Arch Dis Child Fetal Neonatal Ed 2000;82:F182-7.

5. Meek JH, Tyszczuk L, Elwell CE, Wyatt JS. Cerebral blood flow increases over the first three days of life in extremely preterm neonates. Arch Dis Child Fetal Neonatal Ed 1998;78:F33-F7.

6. Osborn DA, Evans N, Kluckow M. Hemodynamic and antecedent risk factors of early and late periventricular/intraventricular hemorrhage in premature infants. Pediatrics 2003;112(1 Pt 1):33-9.

7. Meek JH, Tyszczuk L, Elwell CE, Wyatt JS. Low cerebral blood flow is a risk factor for severe intraventricular haemorrhage. Arch Dis Child Fetal Neonatal Ed 1999;81:F15-8.

8. Hunt RW, Evans N, Rieger I, Kluckow M. Low superior vena cava flow and neurodevelopment at 3 years in very preterm infants. J Pediatr 2004;145:588-92.

9. Rubin JM, Adler RS, Fowlkes JB, et al. Fractional moving blood volume: estimation with power Doppler US. Radiology 1995;197:183-90.

10. Hernandez-Andrade E, Jansson T, Figueroa-Diesel H, Rangel-Nava H, Acosta-Rojas R, Gratacós E. Evaluation of fetal regional cerebral blood perfusion using power Doppler ultrasound and the estimation of fractional moving blood volume. Ultrasound Obstet Gynecol 2007;29:556-61.

11. Rubin JM, Bude RO, Fowlkes JB, Spratt RS, Carson PL, Adler RS. Normalizing fractional moving blood volume estimates with power Doppler US: defining a stable intravascular point with the cumulative power distribution function. Radiology 1997;205:757-65.

12. Heck S, Schindler T, Smyth J, Lui K, Meriki N, Welsh A. Evaluation of neonatal regional cerebral perfusion using power Doppler and the index fractional moving blood volume. Neonatology 2012;101:254-9.

13. Schindler T, Stevenson G, Jayatilake S, Gilbert Y, Oei JL, Welsh A. Reference ranges for neonatal basal ganglia perfusion as measured by fractional moving blood volume. Neonatology 2016;109:91-6.

14. van Bel F, Lemmers P, Naulaers G. Monitoring neonatal regional cerebral oxygen saturation in clinical practice: value and pitfalls. Neonatology 2008;94:237-44.

15. Sortica da Costa C, Greisen G, Austin, T. Is near-infrared spectroscopy clinically useful in the preterm infant? Arch Dis Child Fetal Neonatal Ed 2015;100:F558-61.

16. Counsell SJ, Rutherford MA, Cowan FM, Edwards AD. Magnetic resonance imaging of preterm brain injury. Arch Dis Child Fetal Neonatal Ed 2003;88:F269-74.

17. Kehrer M, Blumenstock G, Ehehalt S, Goelz R, Poets C, Schöning M. Development of cerebral blood flow volume in preterm neonates during the first two weeks of life. Pediatr Res 2005;58:927-30.

18. Takami T, Sunohara D, Kondo A, et al. Changes in cerebral perfusion in extremely LBW infants during the first $72 \mathrm{~h}$ after birth. Pediatr Res 2010;68:435-9.

19. Heymann MA, Payne BD, Hoffman JI, Rudolph AM. Blood flow measurements with radionuclide-labeled particles. Prog Cardiovasc Dis 1977;20:55-79.

20. Logitharajah P, Rutherford MA, Cowan FM. Hypoxic-ischemic encephalopathy in preterm infants: antecedent factors, brain imaging, and outcome. Pediatr Res 2009;66:222-9.

21. Collins SL, Stevenson GN, Noble JA, Impey L, Welsh AW. Influence of power Doppler gain setting on Virtual Organ Computer-aided AnaLysis indices in vivo: can use of the individual sub-noise gain level optimize information? Ultrasound Obstet Gynecol 2012;40:75-80.

22. Sanderson J, Wu L, Mahajan A, Meriki N, Henry A, Welsh AW. Selection of the sub-noise gain level for acquisition of VOCAL data sets: a reliability study. Ultrasound Med Biol 2014;40:562-7.

23. Van der Walt S, Colbert SC, Varoquaux G. The NumPy array: a structure for efficient numerical computation. Comput Sci Eng 2011;13:22-30.

24. Oliphant TE. Python for scientific computing. Comput Sci Eng 2007;9: 10-20. 